\title{
Research on Cold Chain Logistics Distribution Route Based on Ant Colony Optimization Algorithm
}

\author{
Haiou Xiong (iD) \\ College of Port and Shipping Management, Guangzhou Maritime University, Guangzhou 510725, China \\ Correspondence should be addressed to Haiou Xiong; xionghaiou@gzmtu.edu.cn
}

Received 19 November 2020; Revised 2 March 2021; Accepted 24 March 2021; Published 17 May 2021

Academic Editor: Tingsong Wang

Copyright ( $) 2021$ Haiou Xiong. This is an open access article distributed under the Creative Commons Attribution License, which permits unrestricted use, distribution, and reproduction in any medium, provided the original work is properly cited.

\begin{abstract}
The cold chain logistics distribution industry not only demands all goods can be timely distribution but also requires to reduce the entire logistics transportation cost as far as possible, and distribution vehicle route optimization is the key problem of cold chain logistics transportation cost calculation. The traditional optimization method spends a lot of time to search so that it is tough to find the globally optimal path approach, which results in higher distribution costs and lower efficiency. To solve the abovementioned problems, a cold logistics distribution path optimization solution, ground on an improved ant colony optimization algorithm (IACO) is formulated. Specially, other constraints, e.g., the transport time factor, transport cooling factor, and mean road patency factor, can be added to the unified IACO. Meanwhile, the updating mode of traditional pheromone is improved to limit the maximum and minimum pheromone concentration on the road and change the path selection transfer probability. The simulation results and experiment make clear that the IACO algorithm is lower than the chaotic-simulated annealing ant colony algorithm (CSAACO) and the traditional ACO algorithm in terms of convergence speed, logistics transportation distance, and logistics delivery time. At the same time, we have successfully obtained the optimal logistics distribution path, which can provide valuable reference information for improving the economic benefits of cold chain logistics enterprises.
\end{abstract}

\section{Introduction}

With the increasing exploitation of the modern economy, advanced science, and information technology, the existing online shopping paradigm has gradually become an indispensable way of life for people. This shopping mode not only greatly facilitates people's life but also drives the mushroom growth of the emerging logistics industry paradigm. As the "third benefit source," logistics has an increasingly obvious impact on economic development, and more and more enterprises have joined logistics, which has gradually become one of the industries with fierce competition [1-3]. Logistics distribution broadly refers to the logistics campaigns of selecting, processing, casing, partitive, and gathering materials within a certain area according to the needs of users and delivers them to the places designated by users on time $[4,5]$. In the process of logistics and transportation, the research objectives of scholars are to reduce the logistics cost of enterprises, shorten transportation time, further enhance the character of transportation service, and obtain the optimal route of transportation. How to realize scientific logistics distribution is a very complicated and crucial problem that every logistics enterprise must face [6]. Logistics enterprises need to realize the goal of low cost and high efficiency in the transportation process.

As a result of the late start disadvantages of domestic logistics in the current situation, the research time of emerging logistics distribution path design is relatively short. At first, the driver mainly plans the optimal logistics distribution path with his own experience. Due to the lack of scientific guidance, the obtained logistics distribution path is not the optimal one, with low logistics distribution efficiency and high logistics distribution cost.

The key issue to the vehicle routing problem (VRP) lies in the optimization of the whole logistics system [7, 8]. Meanwhile, the problem mentioned above is NP-hard problem [8]. With the increase of quantity distribution of goods, the traditional path planning algorithm is not in a 
relatively short period to get access to the first-rank solution through the system to choose the high-point conveying path distribution vehicle. Optimizing the vehicle scheduling and arranging delivery vehicle distribution order can reduce the distance of the vehicle and the rate of empty. Only when the number of customer nodes and lines is smaller, can the exact solution be obtained. However, when the scale of the problem increases, the exact solution will take a long time and have a low effect. On the other hand, the man-machine interaction method requires managers to have enough professional knowledge of logistics distribution and will increase the randomness of vehicle distribution route selection. Generally, heuristic algorithm should be adopted to solve this kind of problems. This algorithm, referring to the continuous induction, analysis, and reasoning of the experience, has been solved for specific problems, and the method to solve this kind of problems is generated. The goal is to get the satisfactory solution or optimal solution of the global problem with an appropriate cost, which not only saves time but also meets the actual requirements of users. Because of the advantages of high efficiency and simple implementation, heuristic algorithms, e.g., grey wolf optimization (GWO) algorithm [9], chaos particle swarm optimization (CPSO) [10], genetic algorithm (GA) [11], firefly algorithm (FA) [12], and ant colony optimization (ACO) [13], have attracted wide attention in the field of optimization research and developed very fast in the recent years.

It can be seen from the existing studies, considering the ACO algorithm, that there are two problems in the cold chain logistics path scheduling and optimization. On the one hand, there are few studies on the specific application requirements of the ACO algorithm in the cold chain logistics path optimization, ignoring the influence of the algorithm itself on the optimization accuracy and convergence speed. On the other hand, the constraint conditions of path optimal scheduling are too simple to meet the requirements of optimization accuracy under the current complex road conditions. Aiming at the existing problems in the current research, this paper proposes an improved ant colony optimization (IACO) algorithm-based cold chain logistics distribution scheduling and optimization method to reduce cold chain logistics transportation cost and improve transportation efficiency from the perspective of the transport time factor, transport cooling factor, and mean road patency factor.

\section{Related Works}

The ACO is a population intelligent optimization algorithm, which can acquire the typical shortest path between demand nest and food via using the parallel mechanism of positive feedback and the cooperation between ants. This algorithm not only has the advantages of good parallelism and fast solving speed but also has an excellent performance in path optimization and task assignment. Therefore, the ACO algorithm has attracted extensive attention in the field of optimization research and developed very fast in the recent years, from the perspective of medical logistics distribution, agricultural products logistics distribution, port logistics ship transportation path, and supermarket logistics distribution, as well as cold chain logistics distribution.

Based on analyzing the characteristics of medical logistics distribution, the literature [14] put forward reasonable assumptions and constraints of the mathematical model of pharmaceutical logistics and constructed the objective function of optimizing the distribution path. Because of the strong ability to acquire better solutions, the ACO paradigm is utilized to reoptimize the objective function. Simulation results demonstrate the higher efficacy of the proposed scheduling algorithm. With the gradual improvement of residents' requirements for food quality, the literature [15] optimized fresh agricultural products logistics distribution routes, which can save costs and build up the operating potency of relevant enterprises. The first study of fresh agricultural products distribution route optimization model presented VRP and vehicle routing problem with time windows (VRPTW) paradigm then completed the fresh agricultural products logistics distribution path clustering GA optimization design and finally given optimization clustering GA implementation steps. MATLAB was used to complete the experimental design, study the external similarity of fresh agricultural products transportation, analyze the clustering results of logistics distribution and obtaining the path within the group, and conduct the performance test. The results show that this method can scientifically configure the logistics route, step up the number of fresh transport vehicles, and improve the full load rate. After the traditional port logistics ship transportation path optimization, the ship transportation cost is very high and the transportation time is too long. Aiming at the abovementioned problems, a new method of port ship transport path optimization was studied in the literature [16] by using artificial intelligence (AI) algorithm, and the optimization problem was solved through two stages of ship distribution and customer management. The results show that the method based on an AI algorithm can effectively reduce transportation costs and shorten transportation time. Distribution is an important link in logistics, build up the potency of distribution, dramatically reduce logistics costs, and improve the quality of service (QoS). Therefore, it is necessary to choose line optimization. Combined with the characteristics of supermarket logistics distribution, the total transport distance minimum model was established in the literature [17], and simulated annealing (SA) algorithm was used to solve the scheduling problem. The achieved results show that the path scheme was shortened from $131.29 \mathrm{~km}$ of the initial random scheme to $71.294 \mathrm{~km}$ of the optimal solution via the SA algorithm. The path scheme was conducive to reducing the logistics distribution cost and has reference significance for the path selection of supermarket logistics distribution. Aiming at the distribution efficiency and cost of fresh products cold chain logistics distribution, an ACO-based optimization scheduling method for the distribution planning of low carbon cold chain logistics is proposed in the literature [18]. The cold chain logistics model was constructed by adding carbon emission cost and goods damage cost, which considered transportation cost, fixed cost, cold preservation cost, and time scheduling cost. The simulation 
results show that the integration of the GA paradigm and the standard ACO algorithm, the optimization algorithm, has faster convergence speed and higher solution accuracy.

Based on the existing researches, there are two problems in the intelligent logistics path scheduling optimization, considering ACO algorithm: (1) there are few types of research on the specific application requirements of ant colony algorithm taking into the intelligent logistics path optimization consideration, ignoring the clear influence of the scheduling algorithm itself on the optimization accuracy and convergence speed; (2) the constraint conditions for path optimization are too simple to meet the requirements of optimization accuracy under the current complex road conditions. Aiming at the existing problems in the present research, this paper proposes an intelligent logistics distribution path optimization method based on an improved ant colony algorithm.

Aiming at the problems of low solving efficiency and high solving error rate in the current cold chain logistics distribution path scheduling and design methods, to build up the success rate of logistics distribution path formulation as shown in Figure 1, an optimal logistics distribution path design method based on IACO algorithm was proposed. The effectiveness of the IACO algorithm in optimal logistics distribution path design is analyzed through a concrete example, which considers constraints based on transport time, transport cost, refrigeration cost, and average road patency degree.

\section{VRP Problem and Modeling}

VRP is an important research object in the process of cold chain logistics distribution optimization. The vehicle path optimization problem is clearly defined as an NP-hard problem $[7,8]$. With the increasing number of goods distributions, the traditional exact optimization scheduling algorithm and heuristic optimization scheduling algorithm cannot get the optimal yet achievable solution in such a short time. Therefore, the bionic optimization algorithm, regarded as a fast-developing direction in solving combinatorial optimization problems in recent years, is needed to deal with this category of combinatorial optimization paradigm. For example, ACO algorithm is a computer simulation algorithm for biological foraging behavior in nature, which has the features of parallelism, robustness emergence, and evolution, and will become an important method to solve the distribution path problem. Most logistics distribution path scheduling optimization can achieve a certain goal, e.g., the shortest delivery time, the shortest delivery path length, or the lowest delivery cost, and meet some constraints, e.g., the maximum vehicle load, and the end of delivery time. For customers with different distribution points, finding the most scientific and reasonable logistics distribution path includes many key reasons, e.g., distribution center, distribution vehicles, and customer points.

Logistics distribution VRP paradigm can be depicted as follows: considering that a certain number of customer points $N$ each with a different number of goods demand $g_{i}$, a distribution center to provide customers the goods, a vehicle team $M$, is responsible for the goods distribution, capacity of distribution vehicle group $q$ is the appropriate route scheduling, intending to achieve satisfied customers, and certain constraints, e.g., the shortest distance, the smallest cost, and the least time-consuming. VRP involves many elements, the most important of which are target and constraint. The main goals of VRP are to minimize the total transportation cost, decrease the total distribution mileage of distributed vehicles, and minimize the number of distributed vehicles, maximize the level of customer service and other goals. Meanwhile, the constraints of VRP mainly include vehicle capacity constraints, delivery mileage constraints, task time window constraints, vehicle departure time constraints, distribution sequence constraints, the total number of vehicle constraints, vehicle type constraints, return vehicle yard constraints, set delivery type constraints, and customer cargo type constraints. The following assumptions are made in the construction of the mathematical model: distribution center, the location is fixed; there is only one distribution center, which is responsible for all the distributions of vehicle scheduling. Distribution vehicles, onboard capacity, driving speed, and maximum driving distance are the same, and onboard capacity is greater than the customer demand for goods. The distribution vehicles start and end at the same distribution center, and the routes passed by the distribution vehicles cannot be repeated. Customer points, the location coordinates of all customer points, service time, priority, and quantity demanded are known. Therefore, the required number of distribution vehicles is represented as follows:

$$
M=\left[\frac{\sum_{i=1}^{N} g_{i}}{\sigma \cdot q}\right]+1,
$$

where $\sigma$ is a constant depending on constraint condition such as the complexity of goods loading and unloading and varying in the interval of $(0,1)$. In general, the parameter $\sigma=0.85$ is applied in this paper. In the whole model, based on the symbol definition in Table 1 , the costs mathematical model of cold chain logistics distribution path selection is established.

$$
\begin{array}{ll}
\min & C=\sum_{i} \sum_{j} \sum_{k} C_{i j} \cdot Y_{i j k} ; \\
\text { s.t. } \quad & \sum_{i} G_{i} \cdot X_{k i} \leq g_{i}, \quad i=1,2, \ldots, M, \\
& \sum_{i} Y_{k i}=1, \quad i=1,2, \ldots, M, \\
& \sum_{j} X_{k i j k}=Y_{k i}, \quad i=0,1, \ldots, M, \\
& \sum_{i} Y_{k i j k}=Y_{k j}, \quad j=0,1, \ldots, M, \\
X= & X_{i j k} \in S, X_{i j k} \in\{0,1\}, Y_{k i} \in\{0,1\},
\end{array}
$$

where $i$ and $j$ represent the index of the customer point, and $k$ denotes the index of the delivery vehicle. The vehicle $k$ visits the customer $i$ only once, the cargo demand volume of the customer point $i$ is $g_{i}$, and the total amount of customer 


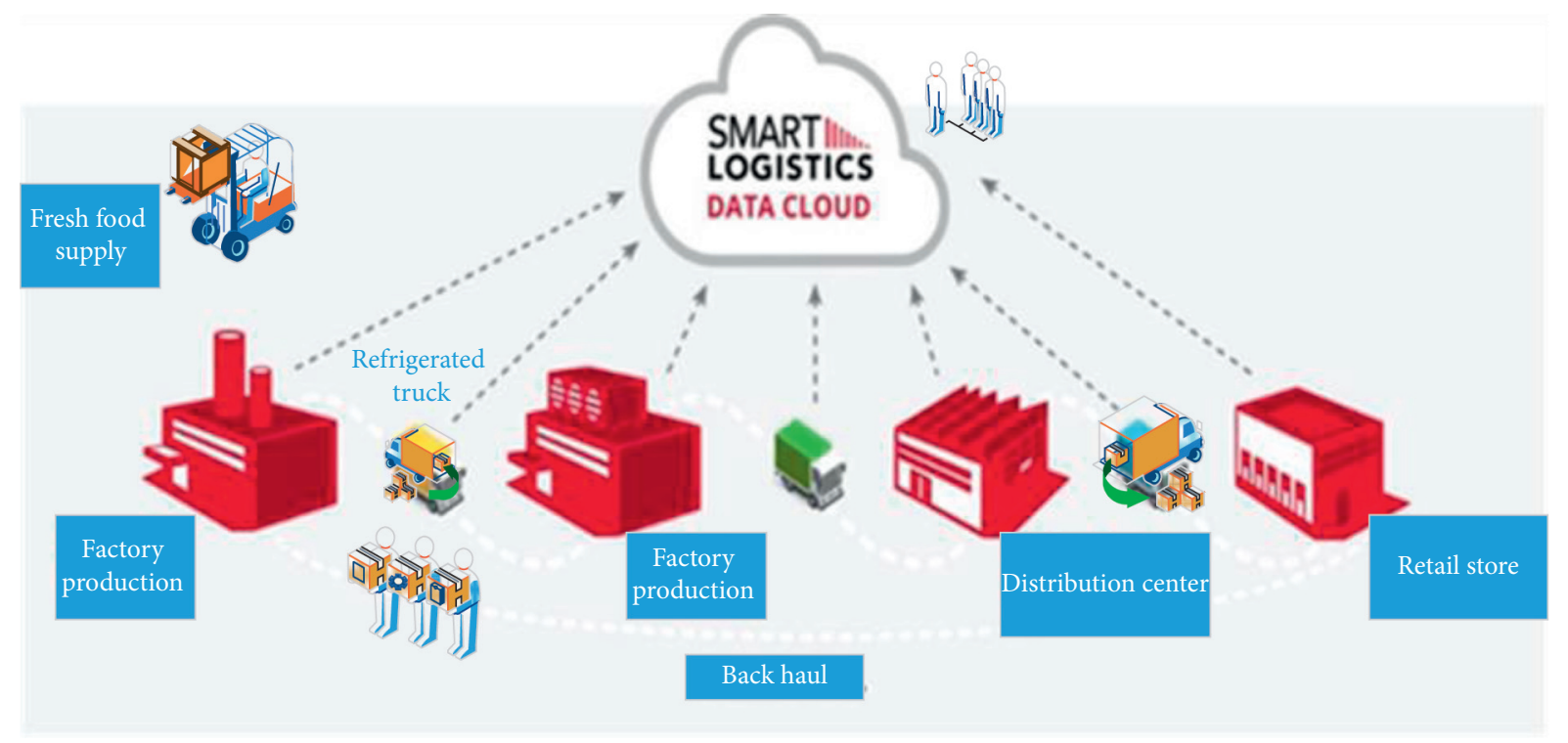

FIGURE 1: Framework of the proposed action quality assessment.

TAble 1: Symbol definition.

\begin{tabular}{l}
\hline Symbo \\
\hline$X_{i k}$ \\
$Y_{i j k}$ \\
$C_{i j}$ \\
$g_{i}$ \\
$G_{i}$
\end{tabular}

demand cannot exceed the maximum capacity of all vehicles in the distribution center. In conclusion, the logistics distribution scheduling and route optimization problem are a typical set of combined optimization paradigm. ACO algorithm is a category of the problem, which can be resolved by the positive feedback and distributed collaborative heuristic search algorithm, and always looks for a source from the food to the nest of the short circuit. This process is quite similar to the logistics distribution route optimization problem. Therefore, to introduce change into the ACO algorithm, resolve it.

\section{Improved Ant Colony Algorithm-Based Optimal Logistics Distribution}

4.1. Traditional Ant Colony Algorithm. The ACO algorithm is regarded as an AI optimization model that simulates the behavior of natural ant colonies in their search for food, which shows that the ant can choose the route according to the pheromone secreted by the preceding ant, and the probability of the route to the food source is proportional to the pheromone intensity secreted on the route. Therefore, a feedback phenomenon of information will be formed in the path of ants, i.e., the more the ants choose a certain path, the more the pheromones are left on the path, and the more likely the subsequent ants will choose this path to find the shortest path.

Through experts researching for years, the application of ACO algorithm has made great research advance and extensive role in various engineering fields. The algorithm with slower speed of convergence is tending to split up into local optimal solution, and other shortcomings can be resolved via the improvement of local pheromone updating rule, dynamic adjustment of related parameters and optimum combination, and global update strategy implementation algorithm optimization to improve the convergence speed of ant colony algorithm, enhance the global search randomness, and significantly inhibit the algorithm appear premature phenomenon.

Suppose in the moment of $t$, the total amount of ants arriving at the node $i$ is $B_{i}(t)$, then the total amount of ants for $n$ nodes (i.e., customer point number) is $m=\sum_{i=1}^{n} B_{i}(t)$. Also, the distance between node $i$ and node $j$ is $d_{i j}$. Initially, all the routes have no ant crawling, and the initial pheromone is the same, namely, $\mu_{i j}=C$, where $\mu_{i j}(t)$ refers to the pheromone concentration on the scheduling route between node $i$ and node $j$ at any time $t$, and $\eta_{i j}(t)$ is the heuristic information function corresponding to the distribution route $R_{i j}$. Then, at the time $t$, the path transition probability of ant $k$ on a node $i$ to the node $j$ is 


$$
P_{i j}^{k}(t)= \begin{cases}\frac{\left[\mu_{i j}(t)\right]^{\alpha} \cdot\left[\eta_{i j}(t)\right]^{\beta}}{\sum_{s \in \text { allowed }(k)}\left[\mu_{i s}(t)\right]^{\alpha} \cdot\left[\eta_{i s}(t)\right]^{\beta}}, & j \in \operatorname{allowed}(k), \\ 0, & \text { other. }\end{cases}
$$

4.2. Improved Ant Colony Algorithm. The prior yet promising ACO paradigm has many advantages, e.g., strong robustness, positive feedback algorithm, and ease to combine with other algorithms. However, the traditional ACO approach also inevitably takes possession of some defects, e.g., it is easy to split up into the local optimal solution, which leads to the phenomenon of search stops, and it needs to search for a long time, when resolving the prior problem of NP-hard, traditional ACO approach for the optimal solution, considering faster speed and higher efficiency, which will be relatively low in the application of traditional ACO paradigm for an optimal path in the process of logistics transportation, and a common method is to solve the cold chain logistics transportation, the shortest distance between the starting point to finish path length is used to measure the merits of the solution. However, in the process of logistics scheduling, it will be an urge to consider the selected road of the average degree of free distribution, e.g., the cost of transportation and shipping time. Therefore, the choice of the road is more than one constraint conditions for the optimal solution of the problem, which considers the standard ACO algorithm, jointly considering multiple constraint conditions of the optimal path selection of IACO, the existing ACO based on heuristic function, and pheromone update methods while using the one node to the endpoint of the IACO inspired by the distance function.

The improved ant colony algorithm enhances the pheromone updating mode by using the constraint function model, which consisted of the material flow transport time factor, the logistics transport cost factor, and the road average mobility factor, so that the logistics transport time is shorter, the transport route is shorter, and the transport efficiency is higher. Heuristic function in traditional ACO algorithm is $\eta_{i j}(t)$, which ignores the ant traverse direction guidance; its value is only considering the reciprocal of the distance between two nodes $1 / d_{i j}$. Because of the ignorance of direction for the inspiration function, it is easily leading to traverse the ants tending to step in the process of the optimization of the shortest and deviate from the direction of the whole optimization, which is limited to the local optimal shortest path rather than the whole. Toward this end, this paper takes into full consideration the distance relationship between the ant's current position, historical position, and future traversal position and improves the heuristic function as follows:

$$
\eta_{i j}(t)=\frac{1}{L_{i}+d_{i j}+d_{j p}}
$$

where $L_{i}$ presents the total length of the path that the ant has traveled to the node $i$. The ant leaves behind pheromones throughout the journey, and when all the ants have completed a round, the ant releases pheromones around the route, depending on the length of the path it has constructed. Therefore, after each round of movement, relevant information brought by ants should be updated. The pheromone update rule of each path can be defined as follows:

$$
\mu_{i j}(t+1)=(1-\rho) \mu_{i j}(t)+\Delta \mu_{i j},
$$

where $\Delta \mu_{i j}=\sum_{k=1}^{m} \Delta \mu_{i j}(k)$ defines the pheromone enhancement located on the scheduling path during this iteration and $\Delta \mu_{i j}(k)$ further defines the pheromone quantity left on the path by the ant $k$ in this iteration. The volatile coefficient $\rho$ is used to describe the persistence of pheromone quantity [13]. Since the fixed value method cannot reflect the characteristics of the ACO algorithm, the adaptive variable value method is adopted in this paper to accelerate the convergence speed and reduce the probability of premature convergence, specifically as follows, where Iteration ${ }_{\text {cur }}$ denotes the number of current iterations and Iteration ${ }_{\max }$ represents the number of maximum iterations:

$$
\rho= \begin{cases}0.2, & \text { Iteration }_{\mathrm{cur}} \in\left[0,0.25 \cdot \text { Iteration }_{\max }\right], \\ 0.4, & \text { Iteration }_{\mathrm{cur}} \in\left[0.25 \cdot \text { Iteration }_{\max }, 0.75 \cdot \text { Iteration }_{\max }\right], \\ 0.6, & \text { Iteration }_{\mathrm{cur}} \in\left[0.75 \cdot \text { Iteration }_{\max }, \text { Iteration }_{\max }\right] .\end{cases}
$$

The optimal solution obtained by the classical optimization algorithm is the local minimum near the given initial value, which is not the minimum from the global point of view. Naturally, it is easy to remind of finding the local minimum through multiple initial points and then finding the global minimum among multiple local minimum values. Based on the process proposed in [13], the optimal path considers the selection of several key factors, e.g., road transport time costs an average of the unobstructed degree to quickly obtain the optimal solution, transport cost factor average road unobstructed degree factor, and then transform the model into the standard ant colony algorithm, and finally achieve the path of the constraint conditions based on realtime updates and dynamic selection, guide choice of logistics transportation toward the optimal path, and get the optimal solution more precisely.

In the analysis of the basic ACO approach, it is found that there are two pheromone updating strategies, i.e., real-time updating and global updating. The former means that the ant renovates the pheromone of the scheduling path immediately after it gets from one node to another. The latter means that the ant updates pheromones along the path only after it has traversed all the nodes. Compared with these two methods, a global updating strategy can accelerate the convergence speed rapidly. At present, many studies have shown that global update has a good effect. Meanwhile, there are some defects. For example, global update in this method usually converges too early, and many ants will quickly converge on the same path so that a better solution cannot be found and obtained, i.e., it falls into the local optimal solution situation. During 
pheromone updating, the system usually only updates the pheromone of the ants that finds the optimal path. The ant pheromone update usually can use the following two ways, one is to find the best performing in the process of circulation of ants; this way is usually slow convergence speed, not too early lead to rapid convergence to a certain path. The ant will continue to find a new path, and it is easier to find a better path. The other way is to find the ants with the best performance in the whole operation. This updating method can rapidly improve the convergence speed and obtain a better solution, but it also prevents the ant colony from searching for a better solution, which makes the entire ant colony easily trapped in a relatively poor path. Therefore, this paper proposes a new hybrid update pheromone strategy, which is in the process of searching previous cycle, using the iterative optimal method of pheromone update in time. This pheromone update is to find out the best in the circle of ants; this method is usually easy to find many more optimal solutions, which can effectively avoid premature of an ant colony in the poor solution. After multiple cycles (in this case, ten cycles) are completed, the updates are then performed using the global optimal solution, i.e., pheromone updates are performed using the ants that have the best performance of the entire operation. After mixing pheromone update rule was adopted, the algorithm will converge to the optimal solution concentration, thus can also find a more feasible solution, and can continue to search for other, more optimal solution and keep the fast convergence speed and can be used effectively to overcome a single global update which is easy to appear prematurely into a locally optimal solution.

4.2.1. Logistics Transport Time Factor, $F_{\text {time }}(j)$. The penalty cost of delivery time is related to the paradigm that whether the delivery vehicle meets the time window of the distribution point. Part of the penalty cost is the loss cost of an early arrival and waiting when the delivery vehicle arrives before the time window required by the distribution point. The other part is when the delivery vehicle arrives after the required time window at the distribution point, resulting in the penalty cost of delay. Then, the time penalty cost is

$$
F_{\text {time }}(j)= \begin{cases}\frac{T(j)}{T_{\max }(j)}, & T(j)<T_{\max }(j) ; \\ 0, & \text { otherwise, }\end{cases}
$$

where $T_{\max }(j)$ represents the upper limit of the maximum estimated time allowed for logistics in road transportation, and $T(j)$ represents the actual time required for logistics transportation. $F_{\text {time }}(j)$ is the factor of logistics transportation time and represents the ratio between the actual transportation time of logistics to the node $j$ and the estimated maximum time. The larger the ratio is, the longer the actual transport time is.

4.2.2. Logistics Transport Cooling Factor, $F_{\text {cool }}(j)$. The main purpose of refrigeration is to maintain a constant temperature to ensure the freshness of fresh products. Suppose that the refrigeration cost mainly includes the cost of keeping the carriage temperature constant in the transportation process of distribution vehicles, the cost of an early arrival and waiting of distribution vehicles, and the cost of the lateness of distribution vehicles. For simplicity, transportation cost is usually related to the price of the product and fuel consumption caused by the multivehicles. Importantly, the longer the vehicle travels, the higher the distribution cost will be. Let the cooling cost be formulated by the ratio of the actual cooling cost $C(j)$ and maximum estimated cooling cost $C_{\max }(j)$ as follows:

$$
F_{\text {cool }}(j)= \begin{cases}\frac{C(j)}{C_{\max }(j)}, & C(j)<C_{\max }(j) ; \\ 0, & \text { otherwise. }\end{cases}
$$

4.2.3. Mean Road Patency Factor, $F_{\text {patency }}(j)$. Traffic performance index (TPD) is an index that comprehensively reflects the unblocked conditions of regional traffic roads $[19,20]$. This index was proposed in the urban road traffic operation evaluation index system, and the ratio between the actual speed and the standard speed is used to reflect the traffic congestion level. The driving speed of the vehicle will be divided according to the TPI standard and the average speed when the road is clear will be the benchmark to calculate the driving speed range of the vehicle. When calculating the driving time of a vehicle, it can be divided into two situations: considering and ignoring the smooth condition of the road. If the smooth condition of the road is not taken into account, it is assumed that the vehicle can keep the standard speed at a constant speed without hindrance, and the driving time can be obtained by comparing the driving distance with the speed. If the road condition is considered, the traveling speed of the vehicle needs to be calculated by TPD to calculate the traveling time. The mean road patency factor $F_{\text {patency }}(j)$ can be represented as follows:

$$
F_{\text {patency }}(j)= \begin{cases}\frac{P(j)}{P_{\max }(j)}, & P(j)<P_{\max }(j) ; \\ 0, & \text { otherwise, }\end{cases}
$$

where $F_{\text {patency }}(j)$ represents the ratio between the patency degree of the route from logistics to the node $j$ and the lowest tolerance of patency degree of vehicle driving road. The larger the ratio in equation (9), the larger probability the distribution vehicle chooses this route. $P(j)$ represents the patency degree of the route to the node $j$, and $P_{\max }(j)$ is the minimum tolerance for road patency of a vehicle. In all, the logistics transport cost factor $F_{\text {cost }}(j)$ can be defined as follows:

$$
F_{\text {cost }}(j)=\omega \cdot F_{\text {time }}(j)+\xi \cdot F_{\text {cool }}(j)+\psi \cdot F_{\text {patency }}(j),
$$

where $\omega, \xi$, and $\psi$ define the weight coefficients of time, cooling, and average road patency in the process of logistics transportation to the node $j$, respectively. To increase the 
speed of convergence for the algorithm, the worst path of the mass path is weakened, a penalty factor is added to reduce the probability of its being selected, and pheromone concentration on the better-quality path is enhanced to make the ant choose the better-quality path. The pheromone updating formula in the standard ACO algorithm based on equation (5) formulation is improved as follows:

$$
\mu_{i j}^{*}(t+1)=\frac{(1-\rho) \mu_{i j}(t)+\Delta \mu_{i j}}{F_{\text {cost }}(j)} .
$$

To build up the convergence speed of this algorithm, the quality path-based worst path is weakened, and the penalty factor is added to reduce the probability of its being selected and increase the pheromone concentration on the stronger and better-quality path leads the ant to choose the betterquality path. The pheromone renovation in standard ant colony algorithm occurs locally. Substituting equations (4) and (11) into equation (3), the path transition probability of ant $k$ on node $i$ to node $j$ for the IACO approach is defined as

$$
\mathrm{P}_{i j}^{k}(t)= \begin{cases}\frac{\left[(1-\rho) \mu_{i j}(t)+\Delta \mu_{i j} / F_{\text {cost }}(j)\right]^{\alpha} \cdot\left[1 / L_{i}+d_{i j}+d_{j p}\right]^{\beta}}{\sum_{s \in \text { allowed }(k)}\left[(1-\rho) \mu_{i j}(t)+\Delta \mu_{i j} / F_{\text {cost }}(j)\right]^{\alpha} \cdot\left[1 / L_{i}+d_{i j}+d_{j p}\right]^{\beta}}, & j \in \text { allowed }(k), \\ 0, & \text { other. }\end{cases}
$$

The steps to solve the optimal logistics distribution path of IACO algorithm are represented in Algorithm 1.

\section{Results and Discussion}

Through the above introduction of algorithm improvement and detailed process, this experiment will use test data to simulate the IACO scheduling paradigm and analyze the test results to evaluate the quality of the improved algorithm for cold chain logistics.

5.1. Environmental Setting. The operating platform of the simulation system is characterized by a CPU capacity of 2.6GMHz, a memory size of $4 \mathrm{~GB}$ and OS mode of Windows 7. To analyze the performance of the optimal cold chain logistics distribution path design method based on the proposed IACO algorithm, considering transport time factor, transport cooling factor, and mean road patency factor, MATLAB software is used to realize the simulation test. The key parameter settings of IACO are interpreted in Table 2.

It is known that a logistics company, only one distribution center, has 8 distribution vehicles, the maximum load resource of each vehicle is $10 \mathrm{~T}$, and the maximum driving distance of each vehicle is $500 \mathrm{~km}$, and it needs to provide resource services for 30 customer points at the same time. The basic information and location of the logistics distribution center and customers are shown in Figure 2.

5.2. Results and Analysis. The proposed IACO algorithm, existing CSAACO algorithm, and traditional ACO algorithm are diffusely used to solve the optimal path of logistics vehicles. Five simulation experiments were conducted to calculate the optimal logistics distribution path length for each experiment, and the results are shown in Figure 3. Each experiment can be conducted under different situations. More specially, the number varying from 1 to 5 represents the customer points varying from 8 to 12 . Besides, it can be seen from Figure 3 that the average length of the optimal logistics distribution path of IACO is $111 \mathrm{~km}$. The ACO algorithm's average length of optimal logistics distribution scheduling path is $114.7 \mathrm{~km}$. Meanwhile, the average length of the optimal logistics distribution path of the CSAACO algorithm is $114.5 \mathrm{~km}$. In addition, the IACO algorithm can obtain a better logistics distribution path, improve logistics distribution speed, reduce the time transmission cost of logistics distribution, and have higher practical application value. As shown in Figure 3, the optimal path length of the IACO algorithm is reduced by $3.7 \mathrm{~km}$ and $3.5 \mathrm{~km}$ compared with ACO and CSAACO, respectively. Importantly, the total number of iterations for each experiment to find the optimal logistics distribution path is represented as follows: IACO: $60,58,52$, 54, 57; CSAACO: 74, 75, 74, 79, 73; ACO: 77, 79, 76, 78, 76.

Compared with the ACO algorithm and the CSAACO scheduling paradigm, the average optimal path time of the IACO paradigm is shortened by $258000 \mathrm{~s}$ and $15000 \mathrm{~s}$, respectively. When customer scale in 100 200, task time of the algorithm for the conventional ACO algorithm, CSAACO algorithm, and the proposed IACO in this paper are not so obvious, with increasing customer scale, under the condition of the scale of performing the same task, the time of the IACO algorithm is far lower than the CSAACO and ACO algorithms. This algorithm can make up for the CSAACO algorithm and the ACO algorithm optimization time longer due to faster speed. Compared with ACO and CSAACO, the path distance of the IACO algorithm is reduced by $25000 \mathrm{~m}$ and $5800 \mathrm{~m}$, respectively. When the customer task scale is 100 , the distance of the proposed algorithm is about $2650 \mathrm{~m}$ shorter than that of the CSAACO algorithm. Compared with the prior ACO algorithm, the scheduling distance of the reformative algorithm is shortened by about $5 \mathrm{~km}$. Moreover, with the increase in scale, the gap is gradually widening. The envisioned IACO algorithm shows more obvious advantages and has better path optimization ability.

The above experimental results make clear that the IACO scheduling paradigm has obvious improvement in optimization efficiency and quality compared with the CSAACO 
Initialize the ant colony, place all ants on the node respectively, and the initial pheromones on all paths are the same;

Iteration times $\mathrm{NC}=0$;

Calculate the probability of each ant choosing the next crawling node, and crawl to the next node according to the calculation results;

Update the pheromone on the path between adjacent nodes;

Update the pheromone on the entire path after all ants crawl the entire path;

Iteration times $\mathrm{NC}=\mathrm{NC}+1$;

Iteration $_{\max }:$ then, output the optimal logistics distribution path;

Algorithm 1: Establishing the optimal logistics distribution path optimization problem corresponding to the directed graph.

TABLE 2: Parameters setting of the IACO approach.

\begin{tabular}{lcc}
\hline Symbol & Description & Value \\
\hline$\alpha$ & 1 & Pheromone factor \\
$\beta$ & 3 & Expected heuristic factor \\
$\rho$ & 0.4 & Pheromone volatility \\
$\omega$ & 0.33 & Weight coefficient of transport time \\
$\xi$ & 0.33 & Weight coefficient of transport cooling \\
$\psi$ & 0.33 & Weight coefficient of mean road patency \\
Iteration $_{\max }$ & 300 & Maximization iterations \\
$N$ & 21 & Total number of customer points \\
$M$ & 8 & Total number of distribution vehicles \\
$q$ & 125 & Total distribution vehicle capacity \\
\hline
\end{tabular}

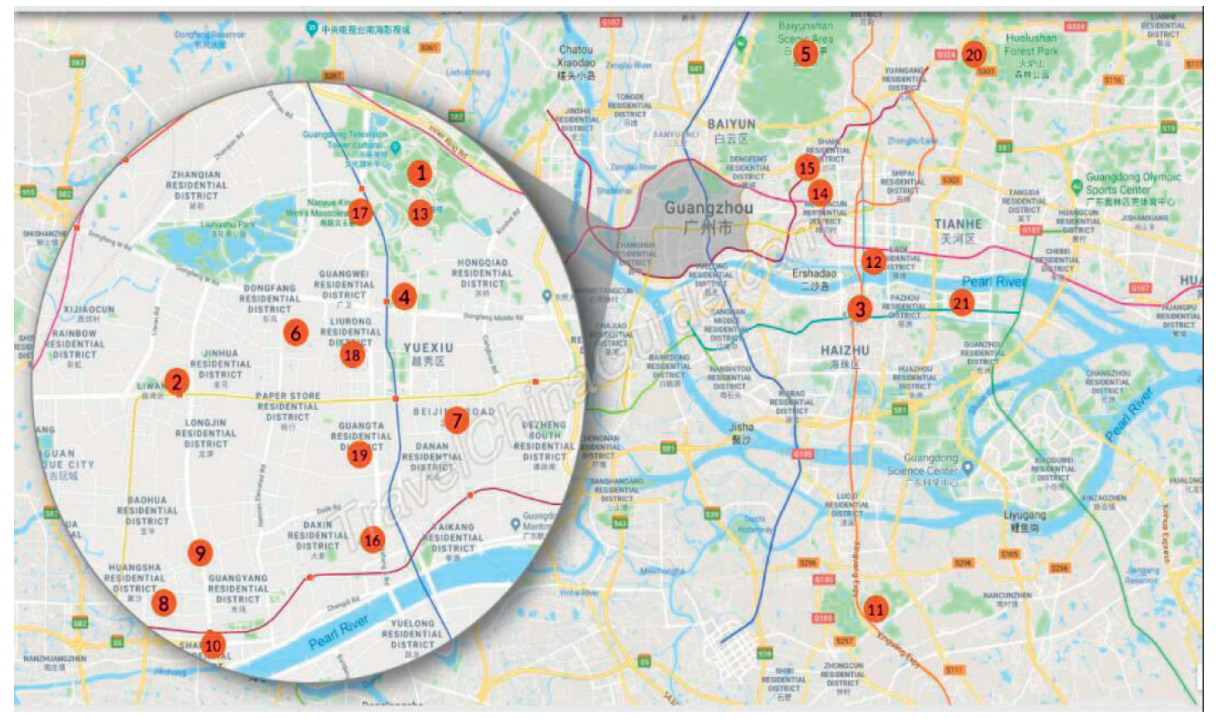

Figure 2: Customer location information map in Guangzhou (brown points).

algorithm and ACO method. Especially as the service customer scale expands unceasingly, the proposed algorithm can more effectively deal with the scheduling problem of logistics distribution time-consuming and poor quality, the advantages are better reflected, the IACO scheduling paradigm is presented in this paper under the condition of the same customer scale task, shorten the time of the distribution tasks effectively, reduce the transportation path distance, accelerate the algorithm convergence speed, and maximize the enhanced efficiency of the logistics industry as a whole and distribution.
As can be seen from Figure 4, the total amount of iterations for the IACO algorithm to find the optimal logistics distribution path was significantly less than that for traditional ACO and CSAACO, which accelerated the solving efficiency of the optimal logistics distribution path. Therefore, the IACO algorithm can be applied to the solution of large-scale logistics distribution path design problems, and its practical application scope is more extensive. Moreover, the convergence speed is much faster, so the path scheduling algorithm in this paper is exceeded by the basic ACO from both convergence speed and the comparison of the results. 


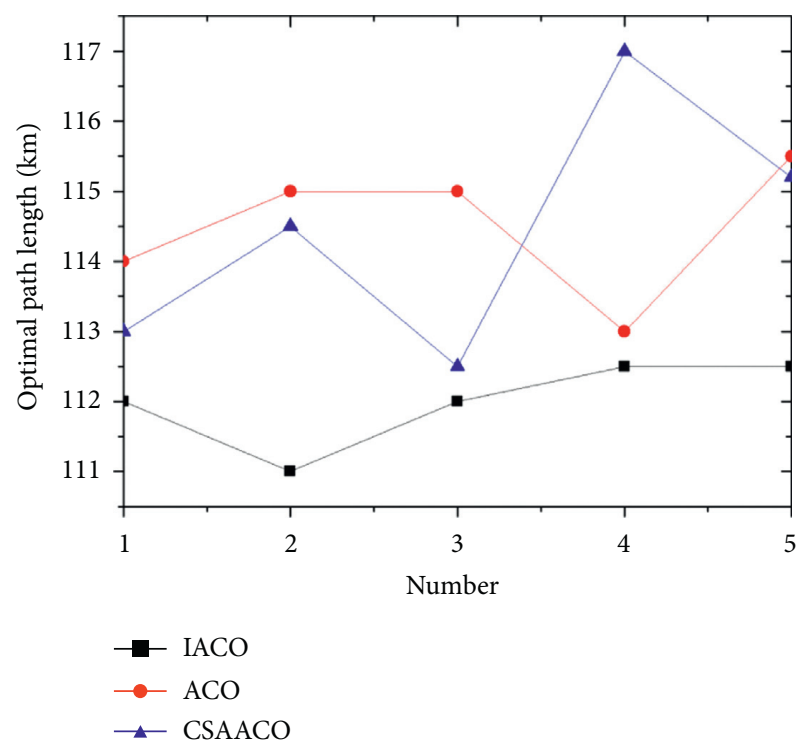

FIgURE 3: The optimal logistics distribution path length comparison.

The problem that the basic ACO is easy to stop at the locally optimal solution is effectively solved in the improved algorithm.

It can be seen in Figure 4 that the IACO scheduling paradigm presented in this paper has great advantages and proves the correctness and effectiveness of the IACO algorithm. This example is solved in the simple case of a single logistics distribution center. If there are multiple logistics distribution centers, it is only necessary to divide the distribution centers into different regions through the decomposition method and then solve them independently. The resulting solution may not be the global optimal solution, but it is still very effective and feasible.

Besides, the total amount of iterations for the IACO scheduling paradigm to find the optimal logistics distribution path is significantly less than that for the CSAACO and ACO algorithm as seen in Figure 4, which speeds up the solving efficiency of the optimal logistics distribution path and can be applied to deal with the design scheduling problem of large-scale logistics distribution path, so the practical application scope is more extensive.

Through the analysis of Figure 5, the appropriate combination of selected parameter values is conducive to obtaining the optimal solution. Therefore, in practical problems, appropriate parameters should be selected according to the actual deployment situation for different problems. To sum up, the optimal search effect was best when $\alpha=1, \beta=3, \rho=0.4$, and the optimal path distance was $880 \mathrm{~km}$.

It can be seen from Figure 5 that the optimal path length IACO algorithm lies in an $880 \mathrm{~km}$, and convergence speed is fast, so both form the convergence speed and carry on the comparison to the final result; this IACO scheduling paradigm is better than the basic ant colony algorithm, which is easy to stall on the local optimal solution of the problem in
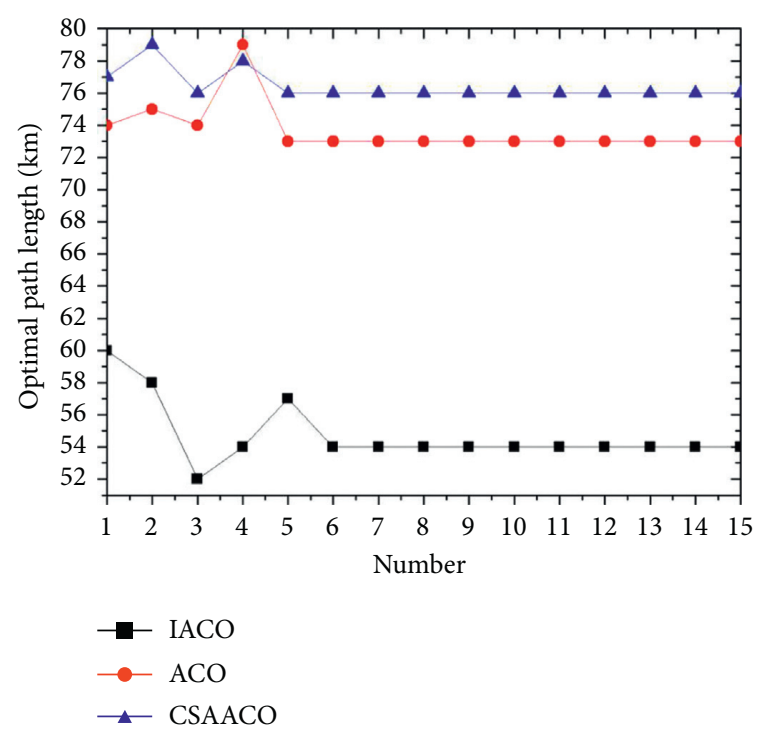

Figure 4: Comparison of iteration times of optimal logistics distribution path.

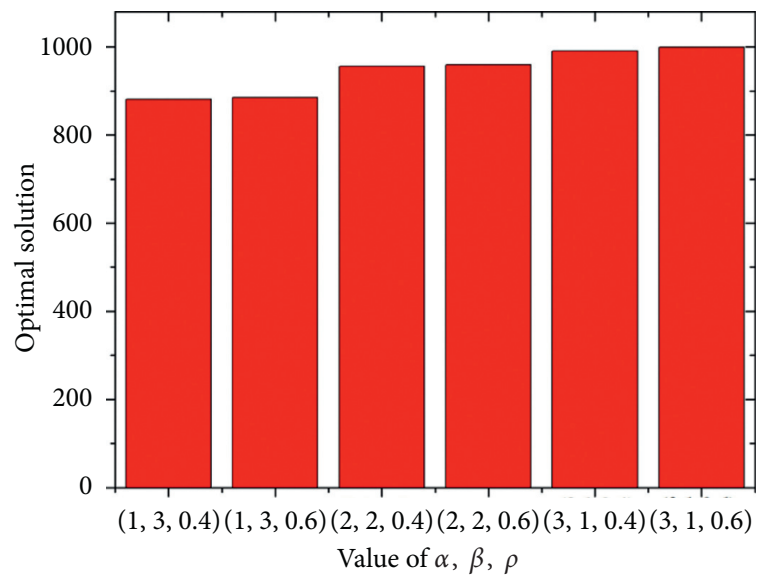

Figure 5: Effect of $\alpha, \beta, \rho$ on path planning.

the improved algorithm which has been effectively solved. As seen from Figure 5, the IACO scheduling paradigm in this paper has great advantages and also proves the correctness and effectiveness of the improved ant colony algorithm.

\section{Conclusions}

The problem of route choice of logistics transportation is crucial to the transportation cost, time, and efficiency of logistics enterprises, and it is a difficult problem faced by all logistics industries. It is of great practical value to analyze the optimal logistics distribution path. To solve some problems existing in the current logistics distribution path, design method is proposed in this paper based on the IACO algorithm of the optimal design method of logistics distribution path, at the same time, to join based on the transport time factor, transport cooling factor, and mean road patency factor, improved pheromone update methods, which 
changed the logistics path transition probability, and compared with the traditional ACO scheduling approach and CSAACO scheduling approach, the outstanding results show that the IACO algorithm can obtain the ideal logistics distribution path, and the search efficiency is high and has a very wide application prospect. Its superior performance is embodied in that it increases the global optimization ability, shortens the distribution path, reduces the cost of distribution for logistics enterprises, improves distribution efficiency, and promotes the rapid development of the logistics industry.

\section{Data Availability}

The data used to support the findings of this study are available from the corresponding authors upon request.

\section{Conflicts of Interest}

The author declares no conflicts of interest.

\section{Authors' Contributions}

H. X. conceptualized the study, developed methodology, utilized software, validated the study, did formal analysis, investigated the study, provided resources, curated the data, wrote the original draft, reviewed and edited the article, visualized and supervised the study, did project administration, and was responsible for funding acquisition. The author read and agreed to the published version of the manuscript.

\section{Acknowledgments}

This work was supported Guangzhou Education Science Planning Project in 2019, "CDIO-Based Research on Innovative and Applied Talent Training Mode" (201912027); Guangzhou Maritime University Innovation and Strength Project in 2018, Logistics Engineering Key Major Construction (F410608); Guangzhou Maritime University Innovation and Strength Project in 2017, Logistics Engineering Key Major Construction (E320103); and Education and Scientific Research Project of Education Department of Guangdong Province in 2020 (2020GXJK403).

\section{References}

[1] S. Winkelhaus and E. H. Grosse, "Logistics 4.0: a systematic review towards a new logistics system," International Journal of Production Research, vol. 58, pp. 18-43, 2020.

[2] L. Rosenbaum, "Facing covid-19 in Italy - ethics, logistics, and therapeutics on the epidemic's front line," New England Journal of Medicine, vol. 382, no. 20, pp. 1873-1875, 2020.

[3] T. P. Vu, D. B. Grant, and D. A. Menachof, "Exploring logistics service quality in hai phong, Vietnam," The Asian Journal of Shipping and Logistics, vol. 36, pp. 54-64, 2020.

[4] K. Zheng, Z. Zhang, and B. Song, "E-commerce logistics distribution mode in big-data context: a case analysis of JD.COM," Industrial Marketing Management, vol. 86, pp. 154-162, 2020.
[5] J. Wang, M. K. Lim, M.-L. Tseng, and Y. Yang, "Promoting low carbon agenda in the urban logistics network distribution system," Journal of Cleaner Production, vol. 211, pp. 146-160, 2019.

[6] X. Cui and C. Xie, "Design and implementation of logistics management system based on SSH technology," Journal of Physics: Conference Series, vol. 1533, p. 042057, 2020.

[7] Z. Wang and J.-B. Sheu, "Vehicle routing problem with drones," Transportation Research Part B: Methodological, vol. 122, pp. 350-364, 2019.

[8] S. Pelletier, O. Jabali, and G. Laporte, "The electric vehicle routing problem with energy consumption uncertainty," Transportation Research Part B: Methodological, vol. 126, pp. 225-255, 2019.

[9] L. Xiao, M. Xu, Y. Chen, and Y. Chen, "Hybrid grey wolf optimization nonlinear model predictive control for aircraft engines based on an elastic BP neural network," Applied Sciences, vol. 9, no. 6, p. 1254, 2019.

[10] Q. Zhang, S. Liu, D. Gong, H. Zhang, and Q. Tu, “An improved multi-objective quantum-behaved particle swarm optimization for railway freight transportation routing design," IEEE Access, vol. 7, pp. 157353-157362, 2019.

[11] Y. Liu, S. Ji, Z. Su et al., "Multi-objective AGV scheduling in an automatic sorting system of an unmanned (intelligent) warehouse by using two adaptive genetic algorithms and a multi-adaptive genetic algorithm," PloS One, vol. 14, p. e0226161, 2019.

[12] X. Zhou, "Path planning of coastal tourism based on the improved firefly algorithm," Journal of Coastal Research, vol. 106, no. sp1, pp. 263-266, 2020.

[13] M. Wang, T. Ma, G. Li, X. Zhai, and S. Qiao, "Ant colony optimization with an improved pheromone model for solving MTSP with capacity and time window constraint," IEEE Access, vol. 8, pp. 106872-106879, 2020.

[14] Z. Liu, Z. Li, W. Chen, Y. Zhao, H. Yue, and Z. Wu, "Path optimization of medical waste transport routes in the emergent public health event of COVID-19: a hybrid optimization algorithm based on the immune-ant colony algorithm," International Journal of Environmental Research and Public Health, vol. 17, no. 16, p. 5831, 2020.

[15] L. Liu, H. Wang, and S. Xing, "Optimization of distribution planning for agricultural products in logistics based on degree of maturity," Computers and Electronics in Agriculture, vol. 160, pp. 1-7, 2019.

[16] L. Kang, "Research on marine port logistics transportation system based on ant colony algorithm," Journal of Coastal Research, vol. 115, no. sp1, pp. 64-67, 2020.

[17] B.-H. Zhou and F. Tan, "A self-adaptive estimation of distribution algorithm with differential evolution strategy for supermarket location problem," Neural Computing and Applications, vol. 32, no. 10, pp. 5791-5804, 2020.

[18] B. Zhao, H. Gui, H. Li, and J. Xue, "Cold chain logistics path optimization via improved multi-objective ant colony algorithm," IEEE Access, vol. 8, pp. 142977-142995, 2020.

[19] R. F. M. Neto, L. E. Paris, F. A. Junior et al., "Environmental performance index for Brazilian public airports: the Infraero experience," Environmental Science \& Policy, vol. 112, pp. 164-171, 2020.

[20] L. Wang, J. Yang, N. Zhang et al., "A spatial-temporal estimation model of residual energy for pure electric buses based on traffic performance index," Tehnicki Vjesnik, vol. 24, pp. 1803-1811, 2017. 